\title{
Near-ultraviolet photodetector based on hybrid polymer/zinc oxide nanorods by low-temperature solution processes
}

\author{
Yun-Yue Lin, ${ }^{1}$ Chun-Wei Chen, ${ }^{1, a)}$ Wei-Che Yen, ${ }^{1}$ Wei-Fang Su, ${ }^{1}$ Chen-Hao Ku, ${ }^{2}$ and \\ Jih-Jen $\mathrm{Wu}^{2}$ \\ ${ }^{1}$ Department of Materials Science and Engineering, National Taiwan University, Taipei 106, Taiwan \\ ${ }^{2}$ Department of Chemical Engineering, National Cheng Kung University, Tainan 701, Taiwan
}

(Received 2 April 2008; accepted 15 May 2008; published online 10 June 2008)

\begin{abstract}
In this article, we have proposed a nanostructured near-ultraviolet photodetector $(<400 \mathrm{~nm})$ based on the $\mathrm{ZnO}$ nanorod/polyfluorene hybrid by solution processes at low temperature. The current-voltage characteristic of the hybrid device demonstrates the typical $p n$-heterojunction diode behavior, consisting of $p$-type polymer and $n$-type $\mathrm{ZnO}$ nanorods, respectively. The relative quantum efficiencies of the hybrid device exhibit a nearly three order difference while illuminated under UV and visible light, respectively. The responsivity for the device can reach to $0.18 \mathrm{~A} / \mathrm{W}$ at $300 \mathrm{~nm}$ by applying a bias of $-2 \mathrm{~V}$, which provides a route to fabricate a low-cost near-UV photodetector. (C) 2008 American Institute of Physics. [DOI: 10.1063/1.2940594]
\end{abstract}

Due to its direct band gap of $3.37 \mathrm{eV}$ and strong exciton binding energy of $60 \mathrm{meV}, \mathrm{ZnO}$-based semiconductors are recognized as very promising photonic materials in the ultraviolet (UV) region. ${ }^{1-3}$ It has been suggested that semiconducting nanowires or nanorods may offer additional advantages for optoelectronic device applications due to the increased junction area, enhanced polarization dependence, and improved carrier confinement in one dimension. Potential applications in many fields such as light emitting diodes, ${ }^{4}$ field effect transistors, ${ }^{5}$ and dye-sensitizer solar cell ${ }^{6}$ have also attracted a great attention recently. Another important application for $\mathrm{ZnO}$ is the UV detection due to its wide band gap and chemical and thermal stability. Until now, UV detectors based on photoconducting layers ${ }^{7}$ or metalsemiconductor Schottky barriers ${ }^{8}$ from $\mathrm{ZnO}$ films have been commonly reported. Since $p$-type doping of $\mathrm{ZnO}$ has remained a challenge, ${ }^{9}$ as a consequence, UV detectors based on $\mathrm{ZnO}$ homojunction or heterojunction structures are still very limited. Compared to most of $\mathrm{ZnO}$ nanostructures which are usually prepared in various gas methods in vacuum and high temperature, ${ }^{10,11}$ much lower temperature deposition methods have been developed recently through solution processes. ${ }^{12}$ In this work, we have developed the simple solution processes to fabricate a near-UV (NUV) detector based on a $p n$-heterojunction consisting of $p$-type polymer polyfluorene (PFO) and $n$-type $\mathrm{ZnO}$ nanorods. The device can be fabricated by preparing the $\mathrm{ZnO}$ nanorods in the much lower deposition temperature regime through solution and utilizing the simple spin-coating method to form the organic/inorganic hybrid thin films.

$\mathrm{ZnO}$ nanorods were grown in electrodeposition from aqueous solution on indium tin oxide (ITO) substrates $\left(15 \Omega / \mathrm{cm}^{2}\right)$ by a two-step electrochemical deposition method in a $0.005 \mathrm{M}$ zinc nitrate aqueous solutions at $95{ }^{\circ} \mathrm{C} .{ }^{13}$ A platinum wire was utilized as the counterelectrode. The potentials of -2.73 and $-2.15 \mathrm{~V}$ relative to the $\mathrm{Pt}$ electrode were applied on the substrates for 1 and $10 \mathrm{~min}$ in sequence during the two-step electrochemical deposition.

${ }^{\text {a) }}$ Author to whom correspondence should be addressed. Electronic mail: chunwei@ntu.edu.tw.
Figure 1(a) shows the typical scanning electron microscope (SEM) image of single crystalline $\mathrm{ZnO}$ nanorods with orientation preferentially vertical to the ITO substrate. The diameter and the length of the nanorods are in the ranges of 80100 and 450-500 nm, respectively. The polymer PFO used in this study was synthesized by using the Suzuki method, ${ }^{14}$ with a molecular weight of $11 \mathrm{KDa}$ and a PDI of 2.07. Figure 1(b) shows the chemical structure of the polymer PFO. The schematic representation of the hybrid $\mathrm{ZnO}$ nanorod/ PFO device structure is shown in Fig. 1(c). A thin PFO layer was deposited to establish a $p$-type contact to the $n$-type $\mathrm{ZnO}$ nanorods by using spin coating. The polymer PFO layer acts as the hole transporting layer of the device, which has a typical hole mobility about $\sim 1.6 \times 10^{-5} \mathrm{~cm}^{2} / \mathrm{V} \mathrm{s} .{ }^{15}$ The effective film thickness of the PFO layer is about $500 \mathrm{~nm}$. The sample was then heated at temperature of $150{ }^{\circ} \mathrm{C}$ for $30 \mathrm{~min}$ to facilitate the infiltration of the polymer into the $\mathrm{ZnO}$ nanorod array. The device was then followed by spin coating a thin layer of poly(3,4-ethylenedioxythiophene)poly(styrenesulfonate) (PEDOT:PSS) with an effective thickness of $50 \mathrm{~nm}$ before thermal evaporation of the Au top electrode. The films were then baked in a vacuum oven for $6 \mathrm{~h}$ at $120^{\circ} \mathrm{C}$. Vapor deposition of the Au top electrode was then carried out at pressure around $2 \times 10^{-6}$ Torr. For the material (a)

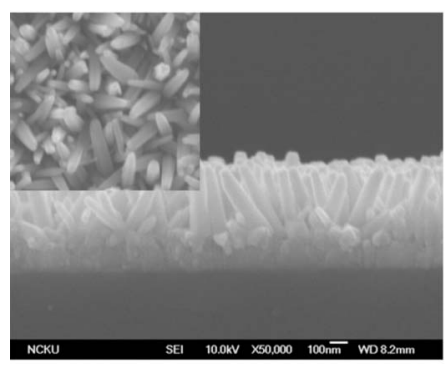

(b)

(c)

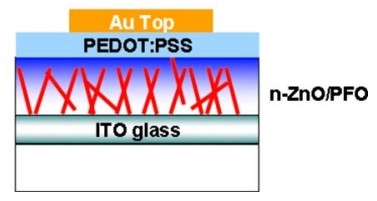

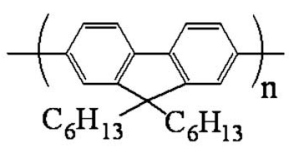

FIG. 1. (Color online) (a) The cross-sectional SEM micrograph of the $\mathrm{ZnO}$ nanorods. The scale bar is $100 \mathrm{~nm}$. The inset shows the top view of $\mathrm{ZnO}$ nanorods. (b) The chemical structure of the PFO polymer. (c) Schematic diagram of the $\mathrm{PFO} / \mathrm{ZnO}$ nanorod hybrid photodiode. 


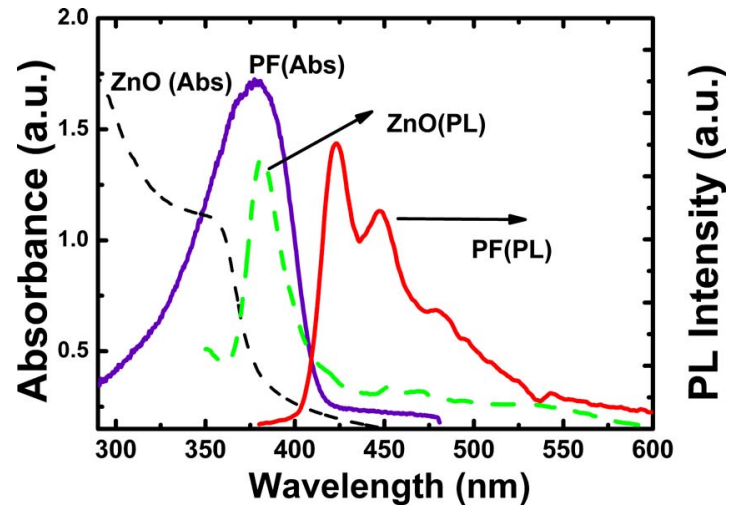

FIG. 2. (Color online) Absorption and PL spectra of $\mathrm{ZnO}$ nanorods (dash line) and polymer PFO (solid line) respectively.

and device characterizations, UV-visible absorption spectra were obtained using an Ocean Optics HR-4000 spectrometer. The steady-state photoluminescence (PL) spectra were measured by using a Perkin-Elmer FS-55 spectrofluorometer. Time-resolved PL (TRPL) spectroscopy was performed with a time-correlated single photon counting spectrometer (Picoquant, Inc.). A pulse laser $(375 \mathrm{~nm})$ with an average power of $1 \mathrm{~mW}$ operating at $40 \mathrm{MHz}$ with a duration of 70 ps was used for excitation. The film thickness was measured by means of the Veeco M6 surface profiler. The current-voltage characterizations (Keithley 2400 source meter) were performed under $10^{-3}$ torr vacuum and under monochromatic illumination at defined beam size (Newport Inc.).

Figure 2(a) shows the absorption and PL spectra of the $\mathrm{ZnO}$ nanorods and polymer PFO used in this study. For the $\mathrm{ZnO}$ nanorods, the fundamental absorption edge is located in the UV region $(<375 \mathrm{~nm})$. The PL spectra exhibit a strong $\mathrm{UV}$ emission peak at about $380 \mathrm{~nm}$, which is related to the near-band edge emission of the wide band gap $\mathrm{ZnO}$. An additional green band at $\sim 450-500 \mathrm{~nm}$ is also observed, which is mainly caused by the intrinsic defects or oxygen vacancies in the $\mathrm{ZnO}$. For the polymer PFO, a broad absorption spectrum ranged from $300-400 \mathrm{~nm}$ with a peak at about $375 \mathrm{~nm}$ is observed. Since PFO has an absorption edge about $3 \mathrm{eV}$, it is also almost transparent in the visible region. In addition, the PL spectrum of PFO shows a broad emission range with two distinct vibronic peaks at 423 and $448 \mathrm{~nm}$, respectively.

Figure 3(a) shows the current-voltage characteristics of the $\mathrm{PFO} / \mathrm{ZnO}$ nanorod heterojunction diode measured in the dark and under illumination at $350 \mathrm{~nm}$, respectively. The typical $p-n$ junction behavior with the clearly rectifying current-voltage $I-V$ characteristics was obtained in the dark, showing a rectification ratio (current ratio measured at +1 versus $-1 \mathrm{~V}$ ) of 122 . This is originated from the formation of $p$ - $n$ junction interfaces between $p$-typed conjugated polymer and the $n$-typed $\mathrm{ZnO}$ nanorods. Under illumination with a $350 \mathrm{~nm}$ light source, a typical photovoltaic characteristic curve can be obtained, with a short circuit current density $\left(J_{\text {sc }}\right)$ of $18 \mu \mathrm{A} / \mathrm{cm}^{2}$, an open circuit voltage $\left(V_{\text {oc }}\right)$ of $335 \mathrm{mV}$, a fill factor of 0.46 , respectively. The dynamic range of the photodiode at zero bias (the current ratio between photocurrent and dark current) is exceeding $10^{4}$. One of the advantages of operating a photodiode under zero or low bias voltage is the low dark current, low noise level, and large dynamic range. The relative quantum efficiencies Downloaded 17 Jun 2008 to 140.112 .32 .21 . Redistribution subject
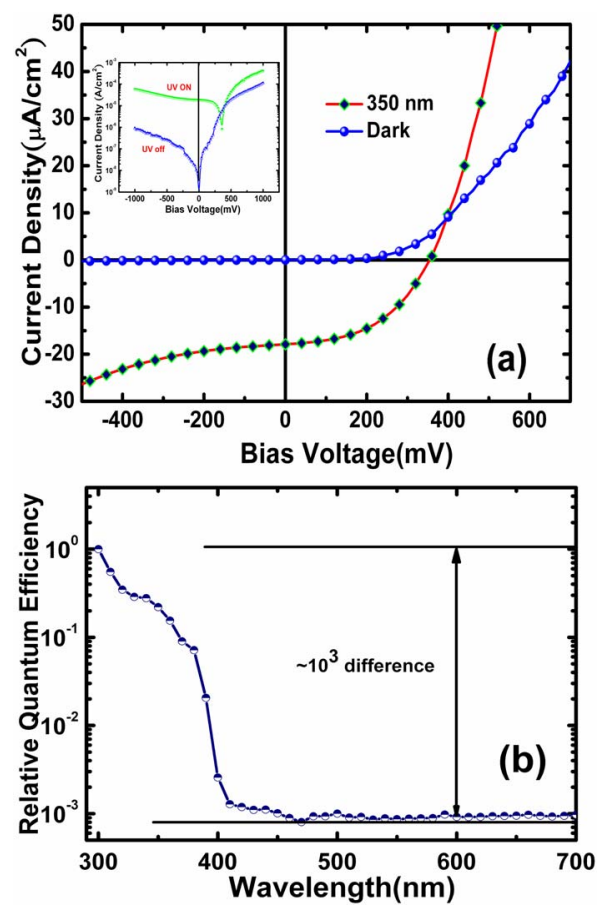

FIG. 3. (Color online) (a) Current-voltage characteristics of PFO/ZnO nanorod heterojunctions in the dark and under $350 \mathrm{~nm}$ illumination respectively. The incident power is $0.69 \mathrm{~mW} / \mathrm{cm}^{2}$. The inset shows the semi-log plot for the above curves. (b) The RQEs for hybrid device. The quantum efficiency under $300 \mathrm{~nm}$ illumination is taken as 1 .

(RQEs) with a spectral range from 300 to $700 \mathrm{~nm}$ for the photodiode based on $\mathrm{PFO} / \mathrm{ZnO}$ nanorod heterojunctions are shown in Fig. 3(b), by taking the quantum efficiency as 1 for the sample under the $300 \mathrm{~nm}$ illumination. The RQEs of the $\mathrm{PFO} / \mathrm{ZnO}$ nanorod hybrid device exhibit a nearly three order difference while illuminated under UV $(300 \mathrm{~nm})$ and visible light $(600 \mathrm{~nm})$, respectively, indicating that the device can be used as a potentially cheap NUV photodetector fabricated from low temperature solution processes.

Figure 4(a) shows the spectral responsivity $S(\lambda)$ of the $\mathrm{PFO} / \mathrm{ZnO}$ nanorod hybrid photodiode operated at different bias conditions. The number of photogenerated electron-hole pairs at the $\mathrm{PFO} / \mathrm{ZnO}$ nanorod interface can be further enhanced by applying a reverse bias to the junction, which may suppress the formation of excitons or decompose excitons into mobile carriers. The responsivity can reach to about $0.18 \mathrm{~A} / \mathrm{W}$ at $300 \mathrm{~nm}$ for the photodiode operated at $-2 \mathrm{~V}$ reverse bias. Due to the large overlap in the UV absorption region between $\mathrm{PFO}$ and $\mathrm{ZnO}$ nanorods, the $\mathrm{PFO}$ thin layer can act not only as the hole transport layer but also as the light absorbing layer. The observed significant increase in $S(\lambda)$ at the wavelengths between 370 and $400 \mathrm{~nm}$ under $-2 \mathrm{~V}$ bias may mainly result from the enhanced charge transfer efficiency from $\mathrm{PFO}$ to $\mathrm{ZnO}$ nanorods. The inset in the Fig. 4(b) exhibits the corresponding electronic energy levels for $\mathrm{ZnO}$ and $\mathrm{PFO}$ obtained from literatures. ${ }^{16,17}$ When the $\mathrm{PFO} / \mathrm{ZnO}$ nanorod hybrid device is illuminated with a wavelength $<350 \mathrm{~nm}$, both $\mathrm{PFO}$ and $\mathrm{ZnO}$ nanorods can be excited. Photogenerated excitons in $\mathrm{ZnO}$ or $\mathrm{PFO}$ can be either recombined radiatively or dissociated from Coulomb attraction at the interfaces between polymer and $\mathrm{ZnO}$ nanorods, by offering an energetically favorable pathway for the electrons (holes) from polymer ( $\mathrm{ZnO}$ nanorods) to transfer onto $\mathrm{ZnO}$ nanorods (polymer). When the device is illumito AIP license or copyright; see http://apl.aip.org/apl/copyright.jsp 

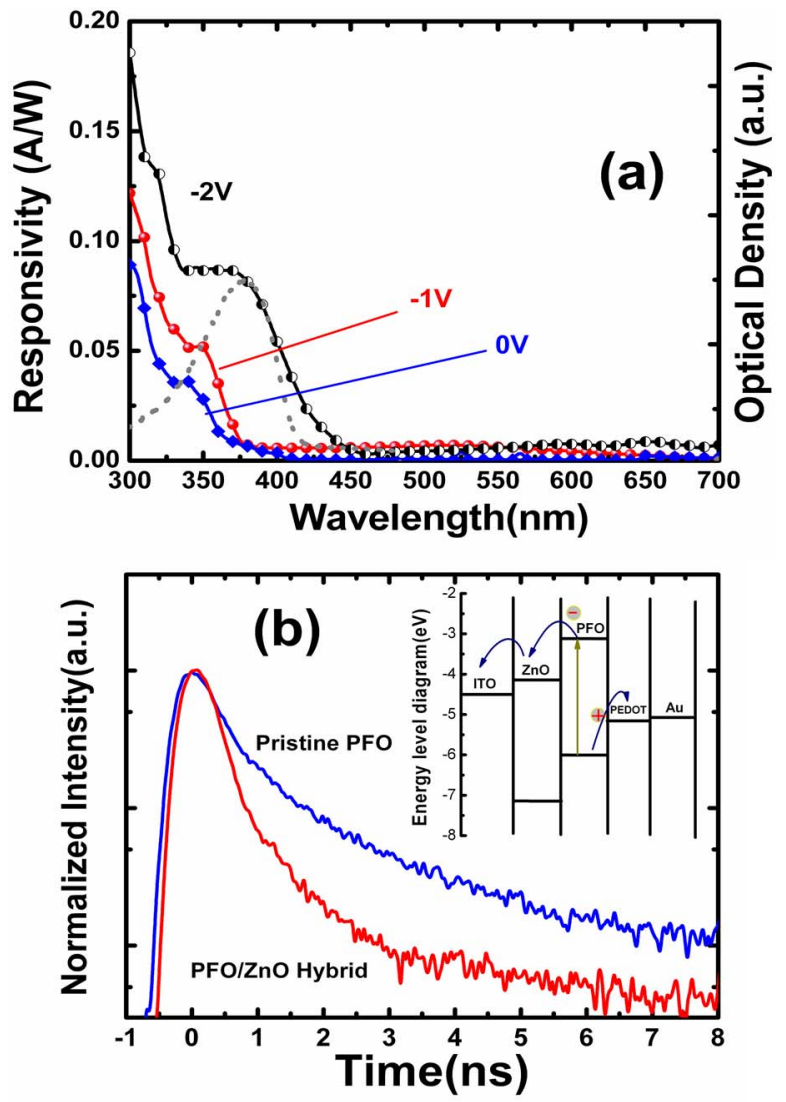

FIG. 4. (Color online) (a) Spectral response of the hybrid photodiode at different biases. The dash line shows the absorption spectra of PFO. (b) The $\mathrm{PL}$ decay curves for the pristine $\mathrm{PFO}$ and $\mathrm{PFO} / \mathrm{ZnO}$ nanorod hybrid thin films, respectively. The exciting wavelength of the pulse is $375 \mathrm{~nm}$ and the PL emission wavelength is probed at $423 \mathrm{~nm}$ of PFO The inset shows the corresponding energy levels for the materials used in the device.

nated with a wavelength between 370 and $400 \mathrm{~nm}$, a large number of excitons are mainly generated from polymer due to the large absorption coefficient of PFO. Figure 4(b) shows the TRPL decay spectroscopy for the pristine PFO and PFO/ $\mathrm{ZnO}$ nanorod hybrid thin films respectively. It is found that the PL lifetime $\tau_{\text {hybrid }}(380 \mathrm{ps})$ for the $\mathrm{PFO} / \mathrm{ZnO}$ nanorod hybrid is much shorter than that of the pristine PFO thin film $\tau_{\text {PFO }}(875 \mathrm{ps})$, suggesting that charge transfer occurs from $\mathrm{PFO}$ to $\mathrm{ZnO}$ nanorods at the interfaces. This accounts for the significant increase in the photoresponse at the wavelength range between 370 and $400 \mathrm{~nm}$, resulting from the efficient charge transfer from $\mathrm{PFO}$ to $\mathrm{ZnO}$ nanorods.

In conclusion, we have reported a $\mathrm{ZnO}$ nanorod/polymer hybrid NUV photodetector using simple low temperature solution processes. We believe this system can be a promising alternative to fabricate the low-cost NUV photodiode since the complete processes are compatible for large area and flexible substrate fabrications.

This work is supported by National Science Council, Taiwan (Project Nos. 96-2112-M-002-030-MY3 and NSC 96-2120-M-001-001).

${ }^{1}$ H. J. Ko, Y. F. Chen, Z. Zhu, T. Yao, I. Kaobayyashi, and H. Uchiki, Appl. Phys. Lett. 76, 1905 (2000).

${ }^{2}$ W. I. Park, Y. H. Jun, S. W. Jung, and G.-C. Yi, Appl. Phys. Lett. 82, 964 (2003).

${ }^{3}$ M. H. Huang, S. Mao, H. Feick, H. Yan, Y. Wu, H. Kind, E. Weber, R. Russo, and P. Yang, Science 292, 1897 (2001).

${ }^{4}$ A. Tsukazaki, A. Ohtomo, T. Onuma, M. Ohtani, T. Makino, M. Sumiya, K. Ohtani, S. F. Chichibu, S. Fuke, Y. Segawa, H. Ohno, H. Koinuma, and M. Kawasaki, Nat. Mater. 4, 42 (2005).

${ }^{5}$ W. I. Park, J. S. Kim, G.-C. Yi, M. H. Bae, and H.-J. Lee, Appl. Phys. Lett. 85, 5052 (2003).

${ }^{6}$ M. Law, L. E. Greene, J. C. Johnson, R. Saykally, and P. Yang, Nat. Mater. 4, 455 (2005).

${ }^{7}$ D. Basaka, G. Aminb, B. Mallikb, G. K. Paulc, and S. K. Senc, J. Cryst. Growth 256, 73 (2003).

${ }^{8}$ S. Liang, H. Sheng, Y. Liu, Z. Huo, Y. Lu, and H. Shen, J. Cryst. Growth 225, 110 (2001).

${ }^{9}$ S. Limpijumnong, S. B. Zhang, S.-H. Wei, and C. H. Park, Phys. Rev. Lett. 92, 155504 (2004)

${ }^{10}$ J. J. Wu, H. I. Wen, C. H. Tseng, and S. C. Liu, Adv. Funct. Mater. 14, 806 (2004).

${ }^{11}$ Z. W. Pan, Z. R. Dai, and Z. L. Wang, Science 291, 1947 (2001).

${ }^{12}$ C. H. Ku and J.-J. Wu, J. Phys. Chem. B 110, 12981 (2006).

${ }^{13}$ J. Cui and U. J. Gibson, J. Phys. Chem. B 109, 22074 (2005).

${ }^{14}$ A. Suzuki and N. Miyaura, Chem. Rev. (Washington, D.C.) 95, 2457 (1995).

${ }^{15}$ R. Pacios, J. Nelson, D. C. Bradley, and C. J. Brabec, Appl. Phys. Lett. 83, 4764 (2003).

${ }^{16}$ M. Graetzel, Nature (London) 414, 338 (2001).

${ }^{17}$ G. Greczynskia, Th. Kugler, and W. R. Salaneck, J. Appl. Phys. 88, 7187 (2000). 